\title{
Applying fragmentation models to the solar atmosphere
}

\author{
J. Ireland ${ }^{1}$, C. A. Young ${ }^{1}$, K. March ${ }^{2}$ and J.-P. Adam ${ }^{2}$ \\ ${ }^{1}$ L3Com/GSI, NASA's GSFC, Mail Code 682.3, Greenbelt MD 20771, USA \\ ${ }^{2}$ Universite de Paris-Sud, 15, rue Georges Clemenceau, 91405 Orsay Cedex, France
}

\begin{abstract}
Recent observational work has shown that the emission in different layers of the solar atmosphere can de described statistically. For example, Pauluhn et al. (2000) show that the frequency distribution of quiet Sun emission in EUV lines is well fit by a lognormal distribution. In addition, Parnell (2002) has shown that the frequency distribution of discrete magnetic elements in the quiet Sun is well fit by a Weibull distribution. These distributions arise naturally from fragmentation studies of materials such as polymers and sediments. It is suggested that fragmentation and its related phenomena may be of use in describing the physics of how the above distributions appear on the Sun.
\end{abstract}

\section{Synopsis}

The studies cited above implicate fragmentation mechanisms in the creation of the observed distributions. Assume that fragmentation is the dominant physical process acting in these observations: from Cheng and Redner (1990), Brown and Wohletz (1995), the equilibrium frequency distribution of particles $n(x)$ of mass $x$ breaking up under external forces is given by

$$
n(x)=\int_{x}^{\infty} n(y) f(x \mid y) d y
$$

where $f(x \mid y)$ is the conditional probability at which $x$ is produced from the breakup of $y$ and contains information on the physical processes acting in the system. Consider now the general solution when $f(x \mid y)=F(x) G(y)$,

$$
n(x)=F(x) \exp \left[-\int^{x} F(m) G(m) d m\right]
$$

Consider $f(x)=f x^{\gamma-1}, g(y)=g$. Then

$$
n(x)=f x^{\gamma-1} \exp \left[-\frac{f g}{\gamma} x^{\gamma}\right]
$$

which is a Weibull distribution. This reproduces the fragmentation solution of Brown and Wohletz (1995) and Parnell (2002). By analogy with Parnell (2002), $x$ is the flux content of a magnetic fragment and $\gamma=0.64(0.59)$ for positive(negative) fragments. The form of $f(x \mid y)$ suggests that the size of the original larger fragment $y$ does not influence the probability of fragmentation, and therefore introduces a condition that could be searched for in numerical simulation of flux tubes.

More generally if, $f(x \mid y) \equiv f(x)$ then

$$
f(x)=n(x) / \int_{x}^{\infty} n(y) d y
$$

and $f(x)$ may be found by through measuring $n(x)$. Schrijver et al. (1997) point out 
that fragmentation is not the only process acting on the flux concentrations at the solar surface. Concentrations of the same polarity are also observed to coalesce. New flux concentrations break the surface continuously, whilst other flux concentrations are cancelled. The model introduced above is an approximation when fragmentation dominates the particular range of fluxes studied. Including other processes in the theoretical description above, coupled with simulation, will lead to a better understanding of the relative importance of the physical processes that create the quiet Sun. Other fragmentation processes are describable using Equation 1.1: consider $f(x)=a x^{\alpha}, g(x)=b y^{\beta}$; then

$$
n(x)=a x^{\alpha} \exp \left[-\frac{a b}{\alpha+\beta+1} x^{\alpha+\beta+1}\right]
$$

so,

$$
f(x \mid y)=a b y^{\alpha-\beta}(x / y)^{\alpha}
$$

In this form, the conditional probability depends on the ratio of the fragment size $x$ to the initial fragment $y$ and the size of the initial fragment.

Consider $f(x)=f$, and some $g(y)$; then

$$
n(x)=C \exp \left[-\int^{x} g(y) d y\right]
$$

The fragmentation here depends purely on the size of the original fragment. Further, if $g(y)=g$, constant, the probability of fragmentation is constant for all fragments and pure exponential distribution results. This has an analogy in reliability modeling where the probability that the lifetime of a component exceeding a time $x$ is $n(x)$ (equation 1.7, suitably normalised) when the probability of failure occurring at any time is constant.

Brown and Wohletz (1995) point out that the lognormal and Weibull distributions are very similar for certain parameter ranges which may lead to the misidentification of distributions. This has implications in diagnosing the fragmentation present, as the distribution shape is used as a strong indicator of the type fragmentation present and the underlying physics. Equations 1.3, 1.5 and 1.7 all arise from considering different fragment size dependent processes, yet result from the solution of the same fundamental equation 1.1. Detailed comparisons of the fit quality for each distribution are required to properly distinguish and diagnose possible fragmentation processes in the data. The quiet Sun is ripe for exploration with such an approach.

\section{Acknowledgements}

This work was funded through NASA contract NAS5-00220.

\section{References}

Brown, W. K., Wohletz, K. H. 1995 J. Appl. Phys. 78(4), 2758-2763.

Cheng, Z., Redner, S 1990 J. Phys. A.: Math. Gen. 23, 1233-1258.

Parnell, C. E. 2002 MNRAS 335, 389-398.

Pauluhn, A., Solanki, S. K., Rüedi, I., Landi, E., Schüle, U. 2000 A. E A. 362, 737-745.

Schrijver, C. J., Title, A. M., van Ballegooijen, A. A., Hagenaar, H. J., Shine, R. A. 1997 Ap.J. 487, 424-436

Wolstenholme, L. C.: Reliability Modelling: A Statistical Approach, Chapman and Hall/CRC, 1999. 